\title{
PANORÁMICA DE LA AUTOTRADUCCIÓN EN GALICIA
}

\author{
Xosé Manuel Dasilva 1 \\ ${ }^{1}$ Universidade de Vigo, Vigo, Galicia, España
}

\begin{abstract}
Resumen: En este artículo se presenta una visión de conjunto acerca de la actividad autotraductora en el espacio gallego. Se presta atención a las fórmulas de los flujos textuales entre gallego y castellano, como la traducción alógrafa, la traducción alógrafa con colaboración del autor, la semiautotraducción y la autotraducción. A continuación se ofrece un resumen de la historia de la autotraducción en Galicia y se indican los motivos que suelen dan lugar a la práctica autotraductora en el caso de los escritores gallegos. Se señala que la característica general de la autotraducción gallega es su naturaleza endógena, lo que suscita tensiones identitarias, lingüísticas y profesionales. Después se determinan como rasgos particulares el predominio de la direccionalidad hacia el castellano, de la simultaneidad de las versiones y de la opacidad de las mismas. Finalmente se pasa revista a algunas categorías concretas, como la retroautotraducción del texto autotraducido, la retraducción del texto autotraducido, la autotraducción del texto traducido y la reautotraducción del texto autotraducido.
\end{abstract}

Palabras clave: Literatura Gallega; Autotraducción; Semiautotraducción; Autotraducción Opaca; Versión Prototípica

\section{AN OVERVIEW OF THE SELF-TRANSLATION IN GALICIA}

Summary: This article presents an overview of the activity of selftranslation in the Galician space. Attention is paid to the formulas of the textual flows between Galician and Castilian, such as the allographical translation, the allographical translation with the collaboration of the author, the semi-translation and the self-translation. Below is a summary 
of the history of self-translation in Galicia and indicate the reasons that often lead to self-driving practice in the case of Galician writers. It is pointed out that the general characteristic of Galician self-translation is its endogenous nature, which generates identity, linguistic and professional tensions. Afterwards, the predominance of directionality towards Spanish, the simultaneity of the versions and the opacity of the same are determined as particular features. Finally, some specific categories are reviewed, such as the retro-self-translation of the self-translated text, the re-translation of the self-translated text, the re-self-translation of the translated text and the re-translation of the self-translated text.

Keywords: Galician Literature; Self-translation; Semi-self-translation; Opaque Self-translation; Prototypical Version

Se aprecian diversos perfiles lingüísticos en lo referente al conjunto de personas que se dedican a la creación literaria en Galicia, sobre todo por la coincidencia de dos idiomas en un mismo territorio. Por una parte, hay autores que desarrollan una actividad monolingüe en gallego o en castellano. Por otra parte, se encuentran en situación distinta los cultivadores a la vez de una y otra lengua, tanto simultáneamente como, en etapas diferentes de su trayectoria, de forma sucesiva. A este segundo grupo corresponde asignar en sentido estricto el sello de escritores bilingües dentro del contexto gallego. Habría que deslindar todavía un contingente reducido integrado por nombres de otras literaturas que ocasionalmente se han servido del gallego de modo alófono. Dos arquetipos sobresalientes son Federico García Lorca, con la obra Seis poemas galegos (Editorial Nós, 1935), y Carles Riba, con los poemarios Cantares d'amor y Cantares d'amigo, datados en 1911, que se recopilaron en el tomo Papers de joventut (Generalitat de Catalunya, 1987).

En Galicia, el creador monolingüe en castellano pocas veces ha evidenciado incertidumbres acerca de su elección idiomática. Por el contrario, quienes utilizan única o preferencialmente el gallego revelan un grado variable de lealtad lingüística. Es posible contraponer, por ejemplo, las actitudes divergentes de Carlos Casares y Alfredo Conde. El primero declaraba sentirse, más que solamente un escritor, representante de una lengua minorizada, y 
por ese motivo, dispuesto a cumplir plenamente con tal compromiso (Carballa 13). El segundo manifestaba, sin embargo, ser un escritor en gallego como otros lo eran en inglés, sin ninguna connotación más allá, por lo que no veía que tuviera que erigirse en una especie de símbolo cívico por trabajar con una lengua no normalizada (Conde, A 254).

Tras algunos debates promovidos en el pasado, hoy en día no admite discusión la exigencia de hacer uso del gallego para poder pertenecer con justo derecho a la literatura gallega. No se acepta el criterio geográfico, aducido en ocasiones pretéritas, según el cual en ella se incluirían Emilia Pardo Bazán, Ramón María del Valle-Inclán, Camilo José Cela o Gonzalo Torrente Ballester, por mencionar autores gallegos significados que escribieron en castellano. Tampoco se reconoce el criterio temático, de manera que obras como Viento del Norte, de la novelista cántabra Elena Quiroga, o Vida e Paixões de Benito Prada, del narrador portugués Fernando Assis Pacheco, serían igualmente ajenas a pesar de abordar asuntos gallegos. El criterio distintivo que prevalece para adscribir a un creador es únicamente el filológico, por lo que solo se tiene en cuenta el empleo del gallego como medio de expresión.

En los autores de Galicia, los transvases textuales entre gallego y castellano son comunes a través de distintas fórmulas, pero domina el desequilibrio como norma general. Resulta más frecuente, objetivamente, que se viertan libros en gallego al castellano que a la inversa. En realidad, desde el centro del Estado español no se tiende a propiciar que los textos en castellano se traduzcan a una lengua periférica. Lo que se demanda es que los textos en gallego se transfieran al castellano, preferentemente a través de autotraducciones. El castellano hasta se convierte en pasarela en las traducciones de una lengua periférica a otra. Versiones gallegas de títulos de Bernardo Atxaga provienen, efectivamente, del texto en este idioma, no del euskera, como Dúas letters (Editorial Galaxia, 1990), Memorias dunha vaca (Ediciones SM, 1998), Un espía chamado Sara (Ediciones SM, 1998), O fillo do acordeonista 
(Edicións Xerais de Galicia, 2004), Obabakoak (Factoría K, 2008) y Sete casas en Francia (Factoría K, 2009).

No debe sorprender, por consiguiente, que las versiones en gallego de productos creados en castellano arrojen un escaso balance en términos globales. Representa poco más que una anécdota, desde luego, que obras en castellano de autores gallegos se trasplanten a la otra lengua. Cuando tal cosa se hace, las fórmulas son la traducción alógrafa y, más raramente, la autotraducción. De lo primero podemos evocar algún título de Camilo José Cela, como A familia de Pascual Duarte (Talleres de Faro de Vigo, 1962), o de Gonzalo Torrente Ballester, como $A$ illa dos xacintos cortados (Ediciós do Castro, 1983). Un prototipo bastante excepcional de autotraductor del castellano al gallego es el narrador bilingüe Carlos G. Reigosa, el cual también se traduce a sí mismo en dirección contraria, aunque con una asiduidad superior. Hay que citar sus autotraducciones al gallego Os outros disparos de Billy (Edicións Xerais de Galicia, 1991) y Oxford, amén (Plans para ninguén) (Edicións Xerais de Galicia, 2005), inicialmente escritas en castellano.

En lo que se refiere a los textos en gallego vertidos al castellano, las fórmulas ordinarias son la traducción alógrafa sin más, la traducción alógrafa con colaboración del autor, la semiautotraducción y la autotraducción. Nosotros separamos, en el interior del terreno tan heterogéneo de la colaboración entre autor y traductor, los conceptos de traducción alógrafa con colaboración del autor y semiautotraducción (Dasilva g, h). Las diferencias residen en que, en el segundo caso frente al primero, consta la admisión expresa de la responsabilidad de la traducción por parte del autor, este propende a imponer sus criterios al traductor y, por último, no es usual que se hagan retraducciones de una versión autotraducida. Dentro de la semiautotraducción, discriminamos cinco modalidades, con sus variantes respectivas: $1^{\circ}$ ) autotraducción en colaboración con un traductor alógrafo; $2^{\circ}$ ) autotraducción revisada por un traductor alógrafo; $3^{\circ}$ ) traducción alógrafa revisada por el autor; $4^{\circ}$ ) autotraducción en colaboración 
con un familiar; y $5^{\circ}$ ) traducción alógrafa de un familiar o un amigo del autor.

Se concluye, así pues, que los textos gallegos se trasladan al castellano básicamente de cuatro modos. El primero es, como decíamos, una traducción alógrafa totalmente ajena al autor. La versión Tic-tac (Ediciones B, 1993), de Suso de Toro, es un ejemplo. El segundo modo estriba en efectuar una traducción alógrafa con colaboración del autor. La colección de relatos ¿Qué me quieres, amor? (Alfaguara, 1996), de Manuel Rivas, encarna un modelo. En tercer lugar, es posible acometer lo que llamamos una semiautotraducción. El texto en castellano de la narración Concubinas (Ediciones Ámbar, 2009), de Inma López Silva, ofrece una muestra de ello. Para finalizar, el cuarto modo traslativo se asienta en la autotraducción propiamente dicha, transponiéndose el autor en solitario. La versión española Ojos de agua (Ediciones Siruela, 2006), de Domingo Villar, suministra una prueba.

En lo que atañe a la participación explícita del autor en las versiones de sus obras, por medio tanto de semiautotraducciones como de autotraducciones, se advierte que se trata de un ejercicio corriente a lo largo de la historia literaria gallega (Dasilva a). En las décadas finales del siglo XIX, en el período denominado Rexurdimento, cuando el cultivo literario del gallego se retoma después de cuatro siglos de casi absoluto mutismo, figuras como Rosalía de Castro, Manuel Curros Enríquez y Eduardo Pondal se aventuran con versiones en la otra lengua de sus propios textos. Hay que referir autotraducciones, por parte de Rosalía de Castro, de los poemas "A ventura é traidora", "A xusticia pola man" y “¡Nin as escuras...!”, todos del libro Follas novas. Curros Enríquez traspasa también composiciones del gallego al castellano, entre ellas “Tempro deserto”, "Sola”, “¡Ai!” y "Cántiga”. Pondal, último miembro de la tríada de grandes autores del Rexurdimento, lleva opuestamente al gallego, en Queixumes dos pinos, textos en castellano de su obra Rumores de los pinos.

Ya en pleno siglo $\mathrm{XX}$, muchos autores gallegos vuelven a traducir personalmente sus textos durante la postguerra. En la 
prosa, Álvaro Cunqueiro despunta con títulos como Merlín y familia (Editorial AHR, 1957), Cuando el viejo Sinbad vuelva a las islas (Argos, 1962), La otra gente (Ediciones Destino, 1975), Tertulia de boticas prodigiosas y escuela de curanderos (Ediciones Destino, 1976) y Las historias gallegas de don Álvaro Cunqueiro (Banco de Crédito e Inversiones, 1981). Su obra Las crónicas del Sochantre (Editorial AHR, 1957) es una supuesta autotraducción, término este que destinamos a la versión elaborada de forma presumible por el autor, por cuanto no se hace referencia a ningún traductor, y se sabe por posteriores noticias que es una traducción alógrafa (Dasilva 112-113e). Más adelante se ha tenido conocimiento, en efecto, de que esta versión de Cunqueiro realmente se debe a Francisco Fernández del Riego.

Eduardo Blanco Amor, otro narrador destacado, también se traduce en La parranda (Compañía General Fabril Editora, 1960), Las musarañas (Editorial Euros, 1975) y Aquella gente (Editorial Seix Barral, 1976). Blanco Amor se responsabilizó en el género teatral de la versión, ahora del castellano al gallego, Farsas para títeres (Ediciós do Castro, 1973). Por aquellos años, en la poesía, el autotraductor más fértil es Celso Emilio Ferreiro, con Cementerio privado (Ediciones Expediente, 1974), Donde el mundo se llama Celanova (Editora Nacional, 1975), El sueño sumergido (Akal Editor, 1975) y Tierra de nadie (Akal Editor, 1975). En el campo narrativo, se hizo cargo de la traducción de El alcalde y otros cuentos (Ediciones Júcar, 1981).

A partir de la restauración de la democracia, el incremento exponencial del número de escritores en gallego conllevó que aumentara sustancialmente la cifra de autotraductores. Debemos hacer referencia a narradores de mayor edad como Marcial Suárez, Xosé Neira Vilas, Carlos Casares, Alfredo Conde, Xavier Alcalá, Carlos G. Reigosa y Víctor F. Freixanes. En generaciones ulteriores se encuadran Manuel Rivas, Suso de Toro, Xosé Carlos Caneiro, Marilar Aleixandre, Domingo Villar, Xurxo Borrazás, Teresa Moure, Ramón Loureiro, Luis Rei Núñez, Xavier Queipo, Antón Riveiro Coello y Miguel-Anxo Murado. En las últimas hornadas 
no ha disminuido ni tan siquiera mínimamente la expansión del fenómeno autotraductor, con nombres como Pedro Feijoo, Ledicia Costas, María Solar, Xulia Alonso, Berta Dávila, Santiago Lopo y Manuel Darriba, entre otros.

En el género poético, la autotraducción se ha multiplicado con una intensidad tampoco desdeñable. De Manuel Rivas son las versiones El pueblo de la noche (Alfaguara, 1997), El pueblo de la noche y mohicania revisitada (Suma de Letras, 2004), La desaparición de la nieve (Alfaguara, 2009) y La boca de la tierra (Visor, 2016). A Iolanda Castaño corresponden El libro de la egoísta (Visor, 2006), Profundidad de campo (Visor, 2009) y La segunda lengua (Visor, 2014). Hace poco salió el volumen bilingüe 13. Antoloxía da poesía galega próxima (Chan da Pólvora Editora - Papeles Mínimos Ediciones, 2017), con jóvenes voces como Oriana Méndez, Celia Parra, Gonzalo Hermo Samuel Solleiro o Antón Blanco que también se traducen.

Un aspecto relevante son los motivos que conducen a los autores a animarse con sus propias traducciones. Resulta indispensable señalar que por lo general obedecen a móviles diversos. Antunes diferenció entre intrinsic reasons y extraneous reasons: las primeras se supeditan a factores personales, mientras que las segundas se relacionan con eventualidades que no están sujetas al control del autor (Antunes 46-47). Tras un análisis centrado particularmente en autotraductores de algunos países, Gentes alegó como causas la existencia de una traducción alógrafa insatisfactoria, la necesidad de acceder a otro mercado que sirva como trampolín y, en fin, el hecho de que la autotraducción no sea por completo independiente del proceso creador (Gentes).

Por nuestra parte, a propósito de los escritores gallegos, una revisión de sus posturas nos ha llevado a aislar motivos variados que no sería costoso identificar en otros sitios (Dasilva b). En primer lugar, está la inquietud experimentada ante la labor de un traductor alógrafo. No es difícil percibir en los autores, además, la idea de que son los traductores en mejor disposición para trasladar sus textos. Tampoco es insólito el deseo de aquilatar en una nueva 
lengua el mérito de la obra. Un motivo más pasa por la intención de adscribirse a otra literatura de resultas de una presunta desatención en la de origen. No se puede olvidar el anhelo de obtener un eco superior merced a una lengua más pujante, así como la ambición de poder dedicarse profesionalmente a la escritura. Finalmente, es visible en ciertas oportunidades la obligación que un autor tiene de traducirse para recalar en otros idiomas

En lo relativo a algunos autores gallegos, la autotraducción ha instigado a menudo preocupaciones traductológicas. Se echa de menos, en todo caso, la reflexión deslizada en el seno de la propia ficción, como no es extraño en otras latitudes, por ejemplo el País Vasco (Manterola Agirrezabalaga 82-83). Eduardo Blanco Amor, uno de los escritores que con mayor clarividencia meditó sobre el acto autotraductor, en la "Justificación" que antepuso a la versión Las musarañas se pronunciaba, desde un ángulo lingüístico, en torno a la profunda disimilitud entre el gallego y el castellano como herramientas de trabajo. Era tajante en cuanto a lo que comportaba saltar "de una lengua acantonada en un finisterre que fue durante siglos una espiritual insularidad", como la gallega, al "striptease suntuoso de esta otra lengua largamente feriada y modelada en el toma y daca de las culturas, con siete largos siglos de hablares y escribires" (Blanco Amor 12a). Álvaro Cunqueiro, en una orientación parecida, explicaba que disfrutaba de mucha más libertad en el idioma propio que en castellano, remarcando que "esta propia anarquía lingüística del gallego, esta falta de normalización, esta capacidad para poder inventar una palabra o un giro son muy importantes" (Conde, P).

Ya en lo alusivo a la estrategia más idónea para encarar el reto autotraductor, Blanco Amor, en el prólogo de la versión Aquella gente (Editorial Seix Barral, 1976), esgrimía que la operación de transportar un texto literario a otro idioma implicaba "deformaciones y licencias", a no ser que la obra en cuestión exhibiera un "contenido mostrenco" o un "estilo convencional" (Blanco Amor 14b). A su entender, dicha circunstancia, ya de por sí embarazosa, se agudizaba en grado notable cuando el traductor 
era el propio autor. Por eso le pedía encarecidamente al lector no ya que le disculpara las "invenciones, que esas son siempre imperdonables", sino que se compadeciera "por el suplicio que supone la autotraducción sin que uno pueda consentirse, en lo posible, la autotraición" (Blanco Amor 15b).

A su vez, Carlos Casares hacía notar, al ser interrogado sobre su versión Los muertos de aquel verano (Alfaguara, 1987), que se había esforzado por evitar transformaciones en el texto autotraducido. Conforme insistía, su empeño se había cifrado en que no se diera en castellano a los lectores un libro alejado del gallego (Moutinho 125). Contrariamente, Suso de Toro aseguraba que, al traducirse, adoptaba más bien el punto de vista del autor que en primera instancia era, no el de un traductor. Añadía que la persona que hace frente a una obra ajena se mueve con unas limitaciones de las que él estaba completamente al margen. De tal manera, si notaba en el proceso de traducción que debía reformar alguna cosa, en todo momento lo hacía con la autoridad que le confería su papel de autor (Giacomel 104-105). Análogamente, Inma López Silva, respecto a la semiautotraducción Los días iguales de cuando fuimos malas (Editorial Lumen, 2017), donde afloran generosos añadidos, sugería que había sido un grato aprendizaje reescribir su propia obra, volviendo a crear mientras se traducía (Velasco Oliaga).

Debe ponerse énfasis en que la característica general de la autotraducción en Galicia es su naturaleza esencialmente endógena, con arreglo a la tipología diseñada por Grutman. La autotraducción endógena es aquella en la que se manejan lenguas que comparten el mismo espacio, en contacto descompensado casi siempre. En contraposición, la autotraducción exógena engloba a los autores en los que la sustitución de la lengua lleva aparejado que se cruce una frontera lingüística, cultural o nacional (Grutman c). En una línea equivalente, Ramis distinguió apropiadamente entre autotraducción intraestatal y autotraducción interestatal (Ramis Llaneras 99-100a; 38-43b).

A tenor de la formulación de Grutman, resulta más que ostensible que la autotraducción del gallego al castellano es ciertamente endógena. Este mismo estudioso planteó otra caracterización provechosa. La 
autotraducción es horizontal entre lenguas de rango semejante, y vertical si afecta a lenguas de rango dispar, pudiendo tratarse en este segundo caso de una supraautotraducción, es decir, desde una lengua de inferior jerarquía a una lengua de superior jerarquía, o de una infraautotraducción, esto es, desde una lengua de superior jerarquía a una lengua de inferior jerarquía. Según esta clasificación, las autotraducciones del gallego al castellano serían verticales y, más concretamente, supraautotraducciones (Grutman d).

La asimetría lingüística que es inherente a la autotraducción desde el gallego al castellano hace que se trate de una operación de signo diglósico, más que puramente bilingüe (Dasilva a). Se detectan múltiples síntomas de tal diglosia, entre los que podemos traer a colación el hecho de que el escritor periférico juzgue que la versión castellana le permite dotar su producción literaria de una resonancia más vasta. Nos hallamos, verdaderamente, ante una diglosia de tipo tanto social como funcional. Por una parte, es social dado que se piensa que el prestigio de la lengua del texto autotraducido favorece el texto en gallego. Suso de Toro se lamentaba de que algunas de sus obras solo hubieran concitado el interés de los círculos locales a partir del momento en que habían sido traducidas al castellano (Navaza and Toro 69).

Por otra parte, la diglosia es funcional porque se reparten las competencias de las dos lenguas, atribuyéndose a la gallega la imagen de doméstica y a la castellana la cualidad de instrumento a fin de alcanzar difusión en otras culturas. Suso de Toro indicaba que se había dado cuenta de que tenía que atravesar el Estado español para arribar a otros países. Hacía hincapié, por ejemplo, en que la autotraducción de Land-Rover había sido determinante para que la obra se trasladara al francés (Pedrós-Gascón 270-271). Al parecer, el editor de la versión en gallego vaticinó que no era una novela que pudiera traducirse. Suso de Toro afirmaría, años más tarde, que cualquier escritor en gallego no tiene capacidad para salir adelante sin un respaldo adecuado desde el centro del Estado. Más aún, sentenciaba taxativamente que, sin mecanismos estatales, ningún autor periférico era viable (Castro). También sin dudarlo, 
Inma López Silva avalaba la misma opinión: "La lengua española es el canal por el que proyectar la literatura gallega hacia todo el mundo (Velasco Oliaga).

La autotraducción endógena ha provocado en Galicia, históricamente, inevitables tensiones de distinta clase. Manuel Curros Enríquez, a finales del siglo XIX, abogaba sin complejos por el uso del gallego frente al castellano. Primeramente, se hacía eco del siguiente preconcepto no poco extendido ya por aquellas fechas: "Se dice comúnmente: escribir en catalán o gallego, pudiendo escribir en castellano para que lo entiendan todos, es una gran prueba de egoísmo". Pero replicaba inmediatamente con energía:

El escritor gallego o catalán no es más egoísta que el alemán o francés, que obliga a que lo traduzcan; y si para traducirlos hay que conocer previamente el lenguaje de la región para la cual han escrito, eso irán ganando la filología y la causa del progreso (Curros Enríquez).

Con el paso del tiempo, los conflictos padecidos por el autotraductor endógeno se han agravado indefectiblemente. Se vislumbra, en primer término, una tensión identitaria, ya que el escritor gallego que se traduce se siente foráneo en el dominio de acogida. Suso de Toro, nuevamente, exteriorizaba que desde el centro del Estado era visto como alguien ajeno, lo que constituía para él un problema de recepción bastante delicado (Pedrós-Gascón 261). Agregaba que no le restaba otro remedio que comprender que no jugaba en su terreno, puesto que se encontraba casi de prestado allí. Solo se aceptaba su presencia dentro de un apartado peculiar que agrupaba a la totalidad de escritores periféricos (Pedrós-Gascón 276). Suso de Toro decía que le adjudicaban, por su procedencia gallega, la etiqueta de integrante de una minoría para la cual únicamente se seleccionaba, como muestra, a unos pocos (Toro 22a). Resumía su experiencia en estos términos: "Ser un autor en lengua gallega en España significa que no saben lo que 
hacer contigo, o que sí saben lo que hacer, meterte en la bodega. Yo me sentí muchos años un polizón a bordo de ese barco" (Pais Beiro). Aún recientemente, Xabier Quiroga, que acaba de editar una traducción alógrafa de su novela Izan o da saca (Edicións Xerais de Galicia, 2015), con el título La casa del nazi (Ediciones B, 2017), aseveraba que se consideraba un exponente del escritor gallego con dificultades para asomarse al exterior (Ocampo).

En el autotraductor endógeno salta a la vista, por otra parte, una tensión lingüística directamente asociada a la identitaria. Suso de Toro, otra vez, exponía que al aterrizar en Madrid con su primer libro traducido al castellano hubo quien no pudo evitar la tentación de formularle la siguiente cuestión sin rodeos: “¿Y por qué no escribes directamente en castellano? Serían libros más universales, llegarían a más lectores" (Toro 24a). Suso de Toro atestiguaba, no sin desesperanza, que "si no haces el camino que hizo Valle-Inclán y tantos otros, escribir en castellano e irte a Madrid, nunca dejarás de ser un extranjero de un país interior pobre y además con acento" (Toro 25a). Álvaro Cunqueiro ya había vivido algo comparable décadas antes. Se quejaba de que se arrinconara las literaturas periféricas tachándolas, displicentemente, de "culturas en lengua vernácula”. Comentaba, con un toque de ironía, a continuación: "A un periodista de Valladolid, que me hacía una entrevista, le pareció mal que yo le dijese que estábamos hablando en su lengua vernácula, el castellano. Creía que esto de vernáculo era como vagón de segunda o tercera clase" (Beneyto 230).

El autotraductor endógeno soporta, en tercer lugar, una tensión profesional. Se observa una batalla persistente entre el autor que escribe en gallego y la dedicación exclusiva a la literatura, algo que demuestra la ausencia de equilibrio entre los dos idiomas que se hablan en Galicia. Álvaro Cunqueiro, en cuya carrera se combinaron los dos códigos por medio de la creación original en gallego y en castellano y de la autotraducción del gallego al castellano, testimoniaba que ejercía como trabajador a tiempo completo en el idioma preponderante, mientras que en gallego no le quedaba otra alternativa que ser un aficionado (Piñeiro). En todo 
caso, no dejaba de preciarse de un firme sentido del deber con relación al gallego, a pesar de ganarse el sustento con el castellano. Proclamaba en cierta ocasión: "Yo soy un escritor profesional que vive de la literatura y tengo que escribir en una lengua que solucione mis problemas, pero tengo el apetito moral e intelectual de escribir en gallego" (Outeiriño).

Cunqueiro se tenía por un escritor bilingüe en la máxima medida. Varias veces contaba que había escrito, cuando era niño, una novela del Oeste donde los vaqueros se expresaban en castellano y los indios en gallego, plasmando así la desigualdad social entre ambas lenguas intuitivamente. Con el transcurrir de los años, Cunqueiro reiteraría el cometido ético que se imponía en lo concerniente a la lengua gallega:

\begin{abstract}
$\mathrm{Al}$ escribir en castellano no siento ninguna responsabilidad en cuanto al status del idioma -sobre su destino, sus problemas literarios, etc. Pero cuando escribo en gallego veo que tengo encima de mis hombros una gran parte de la responsabilidad sobre el presente y el futuro de la lengua de Galicia... (Porcel).
\end{abstract}

Suso de Toro, con relación a su biografía literaria, relataba que se había propuesto ser un escritor en gallego que viviera solamente de la literatura, algo que acabó descubriendo que entrañaba una insalvable contradicción. Durante varios años consiguió sostenerse con sus ocupaciones como escritor, un anhelo que perseguía desde la juventud:

Mi sueño de chaval era escribir una obra literaria a la altura de los autores que admiraba. A partir de un momento, en los años 80 , cuando empiezo a editar, me planteo la posibilidad de ser un escritor que viva de su trabajo, que pueda dedicar su vida a la creación literaria (Pais Beiro). 
No obstante, la implacable realidad terminaría por vencer, incluso tras algunas claudicaciones a las que se vio impelido paulatinamente en menoscabo de su fidelidad hacia la lengua gallega. Al final, a Suso de Toro no le quedó más salida que asumir ese fracaso y regresar a su oficio de profesor de enseñanza secundaria (Castro).

Como consecuencia de las tensiones descritas, cada vez se hace menos inusitado contemplar un proceso evolutivo en algunos escritores gallegos desde el monolingüismo en el idioma propio hasta el monolingüismo en castellano. En tales casos, se entrevén estadios intermedios como la traducción alógrafa, la traducción alógrafa con colaboración del autor, la semiautotraducción, la autotraducción y la creación original en las dos lenguas. La conducta de Alfredo Conde es una clara ilustración de ese proceso, según se desprende de los vaivenes idiomáticos de su itinerario creador. Durante años fue un escritor monolingüe en gallego que se traducía al castellano en persona (Lorenzo Gil). No hace mucho tiempo, sin embargo, optó por abandonar dicho idioma en el plano literario.

El punto de inflexión de esta conversión de Alfredo Conde se localizaría en la novela Huesos de santo (Edhasa, 2010), escrita en castellano, que carece de traducción gallega. En el mismo idioma están redactadas sus dos últimas novelas, Llovida del cielo (Edhasa, 2014) y El beato (Algaida, 2016), esta premiada en el renombrado certamen narrativo Ateneo-Ciudad de Valladolid, autotraducidas luego al gallego como Chovida do ceo (Edicións Xerais de Galicia, 2014) y $O$ beato (Edicións Xerais de Galicia, 2016). Puede conjeturarse que esta suerte de repatriación lingüística de Alfredo Conde tendría su origen en los reveses con los que se tropezó para abrirse camino como autor de éxito en castellano.

Otro ejemplo de un proceso similar es Xosé Carlos Caneiro. Las primeras narraciones de este autor, escritas en gallego, fueron vertidas al castellano por un traductor alógrafo, como se certifica en La rosa de Borges (Espasa Calpe, 2000) y Un juego de apócrifos (Espasa Calpe, 2000). Otra novela suya, Ébora (Espasa Calpe, 2002), la tradujo el propio escritor. Tras esta decisión, entregó a 
la imprenta libros de ficción forjados originalmente en castellano (Rodríguez, X a), como Las arañas en Berlin (Guiverny, 2013) e $Y$ solitario bajo el cielo como siempre estoy yo (Trifolium, 2015).

Dando todavía un paso más, Xosé Carlos Caneiro acaba de publicar Todas las vidas de Madame Bovary (Doña Tecla, 2017), que es la reescritura de una novela casi una década antes editada en gallego, A vida nova de Madame Bovary (Editorial Galaxia, 2008). Otro tanto tiene previsto con una narración editada en esta lengua, Un último destino (Editorial Galaxia, 2010), que próximamente saldrá en castellano (Rodríguez, X b). En su momento, este novelista se había mostrado consciente de que los escritores en gallego se dirigían a un mercado restringido, pero que valía la pena ese sacrificio (Tudela 66).

Aun cuando la característica general de la autotraducción en Galicia es su naturaleza endógena, hay que detallar algunos rasgos más particulares. En primer lugar, si nos fijamos en la direccionalidad, priman abrumadoramente las versiones del gallego al castellano. Grutman reparó en la pertinencia de examinar este ingrediente: “Are the authors constant in their choice of source and target-languages (as with 'regional' writers who translate their work in order to reach a larger audience), or do they freely switch directions?" (Grutman 18b). Parcerisas defendió que la frecuencia aplastante de la unidireccionalidad hacia el castellano procedería justamente de la ausencia de paridad entre las lenguas del Estado español:

C'est cette asymétrie linguistico-culturelle qui fait que les cas d'autotraduction d'une langue de moindre diffusion à une autre langue (dominante, contiguë) de plus grande diffusion se sont multipliés d'une façon spectaculaire (Parcerisas 114).

En lo tocante a la direccionalidad de la autotraducción, una categoría curiosa es lo que designamos como retroautotraducción del texto autotraducido. En ella, el autor materializa una nueva 
versión del texto en la primera lengua, en este caso el gallego, tomando como base el texto autotraducido a la segunda lengua, aquí el castellano. Una muestra es la novela Ambulancia, de Suso de Toro, escrita previamente en gallego (Edicións Xerais de Galicia, 1990), autotraducida seguidamente al castellano (Ediciones B, 2002) y vertida más tarde desde esta lengua al gallego (Edicións Xerais de Galicia, 2004).

Suso de Toro lo hizo así porque había introducido modificaciones trascendentales en el texto de llegada hasta el extremo de rescatar de la muerte, como él mismo confesaba, a un personaje fallecido en la versión gallega: "Y en Ambulancia lo mismo, reescribí, amplié... y hasta resucité a un personaje, el inspector Maquieira. Pensaba en Ambulancia y me parecía que alguien tan canalla y ruin como ese miserable no merecería morir" (Toro b). Suso de Toro valoró que debía reproducir necesariamente tales modificaciones del texto autotraducido en el texto de partida en gallego, emprendiendo una autotraducción de la autotraducción que no es más que una retroautotraducción. La misma maniobra se consumó en otras novelas suyas, como A sombra cazadora (Edicións Xerais, 1994) y Calzados Lola (Edicións Xerais, 1997), con novedades en ediciones sucesivas tras haber sido autotraducidas discrecionalmente.

Un segundo rasgo del quehacer autotraductor en Galicia, ligado a la temporalidad, es el predominio de simultaneous autotranslations o autotraducciones simultáneas, y no de delayed autotranslations o autotraducciones consecutivas, conforme al patrón teórico de Grutman. Las primeras son las versiones que surgen cuando la escritura en la lengua original se halla en curso, mientras que las segundas se generan después de estar rematado el texto de partida (Grutman a, b). Manuel Rivas puntualizaba, hace algunos años, que editaba sus obras en gallego y que durante un tiempo solo se podían adquirir en esta lengua. En el presente, no obstante, los autotraductores gallegos cada vez más lanzan sus libros en los dos idiomas al mismo tiempo.

Tanto es así que no es nada anómalo que los autores escriban a la par en gallego y en castellano, transitando indistintamente de 
una lengua a otra. Para tal opción, Recuenco Peñalver apuntó el nombre autotraducción simultánea bidireccional, que se opondría a la autotraducción simultánea unidireccional (Recuenco Peñalver 205-206). Domingo Villar es un paradigma de esta singular praxis, según ha revelado en bastantes ocasiones. Escribe con antelación en gallego y va traduciendo al castellano normalmente el mismo día, acabando la novela en los dos idiomas porque aprovecha la traducción como procedimiento de corrección (Míguez). Domingo Villar argumentaba que ese modo de autotraducirse respondía a su afán perfeccionista (Faginas), por lo que arrancaba del gallego y se dirigía después a la otra lengua con la finalidad de traducir y retocar paralelamente (Cuíñaz). En una oportunidad más, aclaraba que escribía primero en gallego, corregía, volcaba el texto al castellano, volvía a revisar e incorporaba las rectificaciones de una versión a otra (Rodríguez, S).

Otro rasgo de la autotraducción en Galicia es, en tercer lugar, su apariencia primordialmente opaca, en lugar de transparente, por la forma de presentación ante el público, de acuerdo con la distinción por nosotros propuesta (Dasilva c). En la autototraducción opaca no hay ningún peritexto -cubierta, portada interior, página de créditos, página de los títulos, prólogo o nota introductoria, epílogo, solapas, contracubierta, notas a pie de página, notas finales...- que denote que se trata de una traducción hecha por el autor desde un texto en otra lengua, brindándose así como un original. A nuestro entender, la autotraducción opaca puede ser fortuita o deliberada en consonancia con el nivel de causalidad, y en este último caso voluntaria o forzosa en función de la concurrencia de alguna imposición. Inversamente, la autotraducción transparente sí contiene informaciones en los peritextos que avisan al lector de que tiene en sus manos una obra traducida por el autor. Dependiendo de la consignación más o menos patente y reiterada de la paternidad del autor sobre la traducción, hablaremos de hipertransparencia o hipotransparencia.

Las autotraducciones opacas se hacen progresivamente más profusas en el panorama gallego, siendo ya poco menos que una costumbre la omisión de cualquier noticia acerca de su origen 
en los textos autotraducidos. Podemos escoger al azar ejemplos entre las obras gallegas últimamente traducidas al castellano por sus autores donde esto se verifica, como La venganza del difunto (HarperCollins Ibérica, 2016), de Carlos G. Reigosa, Las horas robadas (Penguin Random House, 2016), de María Solar, o Los hijos del fuego (Ediciones B, 2017), de Pedro Feijoo. Por cierto, las razones de la opacidad no son idénticas en la autotraducción exógena y en la autotraducción endógena. En el primer caso, la opacidad está vinculada casi siempre a la génesis de un nuevo texto con importantes discordancias con relación al texto de partida, por lo cual se estima que posee carácter autónomo. En la autotraducción endógena, sin embargo, tiene que ver con la asimetría del idioma de partida y el idioma de llegada, de manera que suele ser consecuencia de la presión que mantiene la cultura hegemónica sobre la lengua periférica (Dasilva 177f).

La opacidad de la autotraducción endógena produce diversas secuelas, de las que no se escapa la literatura gallega. La primera es un eclipse inicial propiciado por la invisibilidad del autotraductor en cuanto traductor, muchas veces debido a una decisión deliberada más que a un acto fortuito, lo que ocasiona que se desvanezca la pertenencia del texto autotraducido a su cultura genuina. En efecto, tal texto autotraducido opacamente funciona como un original, no como la versión traducida que es en realidad. Esta invisibilidad acarrea un segundo eclipse si las traducciones a otros idiomas se decantan por dicho texto autotraducido como punto de partida.

Otra secuela de la autotraducción opaca es la doble adscripción tanto de los escritores que la practican como de las obras autotraducidas. Ya acabamos de ver que el texto en gallego sufre un doble eclipse: en primer término, al ser traducido de forma opaca desde una lengua periférica, como el gallego, a una lengua central, como el castellano; después, cuando la lengua central actúa de intermediaria para otras versiones. Es posible corroborar lo expuesto en la novela $A$ praia dos afogados (Editorial Galaxia, 2009), de Domingo Villar, autotraducida opacamente al castellano como La playa de los ahogados (Ediciones Siruela, 2009). Las 
versiones en otros idiomas se basaron, sin ninguna excepción, en el texto autotraducido, como la italiana La spiaggia degli affogati (Kowalski, 2010), la alemana Strand der Ertrunkenen (Unionsverlag, 2010), la inglesa Dead on a Galician Shore (Abacus, 2011), la francesa La plage des noyés (Liana Levi, 2011), la polaca Plaża topielców (Wydawnictwo Dolnośląskie, 2011), la neerlandesa Het strand van de verdronkenen (Karakter Uitgevers, 2011), la sueca De drunknades strand (Ekholm \& Tegebjer, 2011) o la portuguesa A Praia dos Afogados (Sextante Editora, 2013).

Conviene resaltar que no solo la opacidad de la autotraducción motiva que las versiones a otras lenguas se lleven a cabo desde el texto autotraducido. En la autotraducción transparente se constata una triple relación jerárquica, que puede también tener influencia, entre el texto primigenio, rótulo este que preferimos en vez de texto original para no dirimir la primacía de cada uno de los textos (Dasilva 63c), y el texto autotraducido: $1^{\circ}$ ) superioridad del texto primigenio; $2^{\circ}$ ) igualdad del texto primigenio y el texto autotraducido; y $3^{\circ}$ ) superioridad del texto autotraducido. La primera situación ocurre cuando el autor reserva la condición de original para el texto primigenio, mientras que la segunda aparece si el autor se inclina por una posición ambivalente.

El último supuesto, donde el autor antepone el texto autotraducido al texto primigenio, acostumbra a darse cuando este dictamina que el primero es una versión prototípica que debe preferirse para la traducción a otros idiomas (Dasilva j). A nuestro juicio, se concede la categoría de versión prototípica al texto autotraducido por dos razones, que se manifiestan juntas o por separado. En primer lugar, porque el autotraductor, luciendo el rol de autor, ha intercalado alteraciones de orden estético, más que traductológicas, en el texto autotraducido. En segundo término, debido a que el autotraductor, en calidad de traductor, se ha encargado de resolver en el texto autotraducido los obstáculos lingüísticos y culturales del texto primigenio, con el fin de hacer menos complicada la traducción a las restantes lenguas.

La existencia de un texto autotraducido como versión prototípica repercute de distintas maneras a la hora de trasladar la obra a otros 
idiomas. Una demostración es lo que sucede con la narración Merlín e familia $i$ outras historias (Editorial Galaxia, 1955), de Álvaro Cunqueiro, puesta en castellano por él mismo como Merlín y familia (Editorial AHR, 1957). El autor perpetró en el texto autotraducido abundantes cambios microtextuales en forma de adiciones, supresiones y permutaciones. Además, desde el punto de vista macrotextual, insertó en la obra materiales inéditos que revisten un incuestionable alcance (Dasilva i).

Todo esto determina que se pueda dudar de la supremacía del texto primigenio en gallego al elegir la fuente para las versiones en terceros idiomas, hasta el punto de que no estaría fuera de lugar calificar el texto autotraducido de versión prototípica. Así, aunque algunas traducciones de la obra de Cunqueiro se fundamentaron en el texto primigenio en gallego, como la asturiana Merlín y familia y otres histories (Llibros del Pexe, 1990) y la brasileña Merlim e família e outras estórias (Mar Maior, 2015), otras optaron por valerse del texto autotraducido en castellano, como la alemana In Gesellschaft des Zauberers (Insel Verlag, 1992). Se da la circunstancia de que en más versiones, como la inglesa Merlin and Company (Everyman, 1996) y la italiana Merlino e famiglia (Miranda) (Morlacchi Editore, 2006), se operó conjuntamente con el texto primigenio y el texto autotraducido, lo que dio como resultado una traducción compilada.

Antes de poner fin a este trabajo, nos gustaría hacer alusión, aunque sea concisamente, a algunas categorías específicas que depara la autotraducción en Galicia. Además de la retroautotraducción del texto autotraducido, vista ya anteriormente, hay que llamar la atención sobre lo que llamamos la retraducción del texto autotraducido, que consiste en transferir por parte de un traductor alógrafo un texto traducido a la misma lengua por el autor (Dasilva d). Cabe recordar la serie de relatos Xente de aquí e de acolá (Editorial Galaxia, 1971), de Álvaro Cunqueiro, trasladada como La otra gente (Ediciones Destino, 1975) por él mismo y, algunos años más tarde, vertida con el título Gente de aquí y de más allá (Iberia, 1990) por Basilio Losada. 
Otra categoría reseñable viene proporcionada por la autotraducción del texto traducido, que se da cuando un autor decide transvasar una obra suya llevada antes por un traductor alógrafo a ese idioma. De esto es ejemplo Xavier Alcalá, quien corrió con el desafío de poner en castellano una obra que estaba en esta lengua gracias a otro traductor. Así se comprueba en la novela Nos pagos de Huinca Loo (Ediciones Nós, 1982), transvasada por un traductor alógrafo como Cristiano muerto (Ediciones Nós, 1983) y autotraducida con posterioridad como Huinca Loo. Un arroyo en la frontera (Mar Maior, 2016).

Una cuarta categoría es la reautotraducción del texto autotraducido, la cual se cumple si un autotraductor resuelve afrontar una nueva versión de un texto que había traducido él mismo. Se puede traer a la memoria el caso de Xosé Neira Vilas, quien optó por actualizar la traducción al castellano de Memorias dun neno labrego (Editorial Follas Novas, 1961), titulada Memorias de un niño campesino (Ediciones Júcar, 1974), décadas más tarde (Kalandraka, 2009). Manuel Rivas hizo algo equiparable con la novela En salvaxe compaña (Edicións Xerais de Galicia, 1993), cuya primera versión en castellano, En salvaje compañía (Alfaguara, 1994), revisó años después (Alfaguara, 2004).

No deseamos concluir sin resaltar que Galicia constituye un marco ideal, por la abundancia y la variedad de la práctica autotraductora, para aproximarse especialmente a la dinámica de la autotraducción endógena. En efecto, es un entorno extraordinario que permite ahondar, desde una perspectiva fecunda, en los problemas derivados de la convivencia desajustada de dos lenguas en una misma comunidad, con el objeto de interpretar la función que le corresponde a la autotraducción en tal escenario. Además, no se debe dejar de poner de relieve que, al margen de la mera descripción, a partir de esta área concreta se pueden abstraer conceptualizaciones sugestivas, las cuales son de aplicación fructífera en otros lugares.

Cad. Trad., Florianópolis, v. 39, n 3, p. 291-316, set-dez, 2019. 


\section{Referencias}

Antunes, Maria Alice. "The decision to self-translate, motivations and consequences: a study of the cases of João Ubaldo Ribeiro, André Brink and Ngugi wa Thiong'o". In L'Autotraduction aux frontières de la langue et de la culture. Limoges: Éditions Lambert-Lucas (2013): 45-52.

Beneyto, Antonio. Censura y política en los escritores españoles. Barcelona: Editorial Euros, 1975.

Blanco Amor (a), Eduardo. "Justificación”. In: Blanco Amor, Eduardo. Las musarañas. Barcelona: Editorial Euros (1975): 7-13.

Blanco Amor, Eduardo (b). “Justificación”. In: Blanco Amor, Eduardo. Aquella gente. Barcelona: Editorial Seix Barral (1976): 9-17.

Carballa, Xan. “Carlos Casares”. In: Carballa, Xan. A Nosa Terra 720 (1996): 12-13.

Castro, Rocío. “A volta de Suso de Toro”. In: Rocío, Castro. Sermos Galiza, 8-VI-2017.

Conde, Alfredo. “Autopoética”. Boletín Galego de Literatura 15-16 (1996): 253-254.

Conde, Perfecto. “Álvaro Cunqueiro: la tristeza es un lujo para jóvenes”. El País, 20-IV-1980.

Cuíñas, Teresa. “Un supervendas galego é posible”. El País, 25-IX-2009.

Curros Enríquez, Manuel. "Caldo de grelos, por Armada Teijeiro". Tierra Gallega, 29-IX-1885.

Dasilva, Xosé Manuel (a). “Autotraducirse en Galicia: ¿bilingüismo o diglosia?”. Quaderns: Revista de traducció 16 (2009): 143-156. 
Dasilva, Xosé Manuel (b). "La autotraducción vista por los escritores gallegos". In: Traducción y autotraducción en las literaturas ibéricas. Bern - Berlin: Peter Lang (2010): 265-279.

Dasilva, Xosé Manuel (c). "La autotraducción transparente y la autotraducción opaca”. In Aproximaciones a la autotraducción. Vigo: Editorial Academia del Hispanismo (2011): 45-68.

Dasilva, Xosé Manuel (d). "La retraducción del texto autotraducido. Xente de aquí e de acolá, de Álvaro Cunqueiro". In L'Autotraduction aux frontières de la langue et de la culture. Limoges: Éditions Lambert-Lucas (2013): 251-259.

Dasilva, Xosé Manuel (e). Estudios sobre la autotraducción en el espacio ibérico. Bern: Peter Lang, 2013.

Dasilva, Xosé Manuel (f). "La opacidad de la autotraducción entre lenguas asimétricas”. Trans. Revista de traductología 19, 2 (2015): 171-182.

Dasilva, Xosé Manuel (g). "En torno al concepto de semiautotraducción". Quaderns. Revista de traducció 23 (2016): 15-35.

Dasilva, Xosé Manuel (h). "La traducción alógrafa con colaboración del autor frente a la semiautotraducción: João Guimarães Rosa como modelo". Romance Notes 57, 1 (2017): 121-131.

Dasilva, Xosé Manuel (i). “Álvaro Cunqueiro, autotraductor. Merlín y familia como versión prototípica”. Iberoromania 85 (2017): 18-32

Dasilva, Xosé Manuel (j). "La autotraducción como versión prototípica". Meta. Journal des Traducteurs. Translator's Journal LXIII, 1 (2018): 234-251.

Faginas, Sandra. "Domingo Villar. Autor dos best-seller Ollos de auga e A praia dos afogados". La Voz de Galicia, 26-XII-2009.

Gentes, Eva. “... et ainsi j'ai décidé de me traduire. Les moments déclencheurs dans la vie littéraire des autotraducteurs". In: Ferraro, Alessandra and Grutmann, 
Rainer. (Eds). L'Autotraduction littéraire: perspectives théoriques. Paris: Classiques Garnier (2016): 85-101.

Giacomel, Giada. La autotraducción entre castellano y gallego: "A esmorga" y "La parranda" de Eduardo Blanco Amor, obras en comparación. Padova: Università degli Studi di Padova, 2015.

Grutman, Rainier (a). "Brian T. Fitch, Beckett and Babel: An Investigation into the Status of the Bilingual Work". Target. International Journal of Translation Studies 3, 1 (1991): 124-127.

Grutman, Rainier (b). "Auto-translation”. In: Baker, Mona. (Ed). Routledge Encyclopedia of Translation Studies. London - New York: Routledge (1998): 1720.

Grutman, Rainier (c). "L'écrivain bilingue et ses publics: une perspective comparatiste". In Écrivains multilingues et écritures métisses. L'hospitalité des langues. Clermont Ferrand: Presses Universitaires Blaise Pascal (2007): 31-50.

Grutman, Rainier (d). "L'autotraduzione verticale ieri e oggi (con esempi dalla Spagna cinquetencesca e novecentesca)". In: Árquez, Marcial Rubio and D'Antuono, Nicola. (Eds) Autotraduzione. Teoria ed esempi fra Italia e Spagna (e oltre). Milano: Edizioni Universitarie di Lettere Economia Diritto (2012): 33-48.

Lorenzo Gil, César. "Alfredo Conde: Son un escritor sen parroquia”. Biosbardia, 7-II-2017.

Manterola Agirrezabalaga, Elizabete. "La autotraducción en el contexto vasco: entre distancia interlingüística y la constitución de un campo literario nacional transfronterizo". Glottopol 25 (2015): 71-87.

Míguez, María. "Domingo Villar: Es más fácil entender la historia de España leyendo a Vázquez Montalbán”. El Mundo, 4-X-2010.

Moutinho, José Viale. “A alegría de o escoitar”. In: Carballa, Xan and Villalaín, Damián. (Eds). Carlos Casares. Os amigos, as imaxes, as palabras. Vigo: Promocións Culturais Galegas - Fundación Carlos Casares (2004): 119-128. 
Navaza, Gonzalo e Toro, Suso de. "A creación literaria como provocación". Tempos Novos 48 (2001): 66-72.

Ocampo, Elena. “Xabier Quiroga, escritor”. Faro de Vigo, 22-VII-2017.

Outeiriño, Maribel. "Álvaro Cunqueiro: Soy un antimarxista visceral”. $L a$ Región, 8-VII-1979.

Pais Beiro, Mario. "Suso de Toro: España vive una crisis nacional porque no tiene una cultura nacional”. eldiario.es, 24-X-2014.

Parcerisas, Francesc. "Idéologie et autotraduction entre cultures asymétriques". Atelier de Traduction 7 (2007): 111-119

Pedrós-Gascón, Antonio. Conversas con Suso de Toro. Como saba de liño. Vigo: Edicións Xerais de Galicia, 2005.

Piñeiro, Alfonso. "Charla con Álvaro Cunqueiro”. El Adelanto, 16-IV-1975.

Porcel, Baltasar. "Álvaro Cunqueiro, un hombre de nación gallega”. Destino, 8-III-1969.

Ramis Llaneras, Josep Miquel (a). "La autotraducción y el difícil encaje de sistemas literarios en contacto", Eu-topias 5 (2013): 99-111.

Ramis Llaneras, Josep Miquel (b). Autotraducció. De la teoria a la pràctica. Vic: Eumo Editorial, 2014.

Recuenco Peñalver, María. "Más allá de la traducción: la autotraducción". Trans. Revista de traductología 15 (2011): 193-208.

Rodríguez, Salvador. "Domingo Villar, un Vigo de cine". Faro de Vigo, 4-XII2015. 
Rodríguez, Xosé Manoel (a). "Xosé Carlos Caneiro, escritor: É un acto de rebeldía, quería reinventarme”. La Voz de Galicia, 8-XII-2013.

Rodríguez, Xosé Manoel (b). "Xosé Carlos Caneiro: Escribo novelas femininas e feministas, ese é o meu universo". La Voz de Galicia, 6-III-2017.

Toro, Suso de (a). “Mi pregunta mágica: ¿escribir en gallego”. Galicia 669 (1996): 22-25.

Toro, Suso de (b). "La traducción: todo un milagro”. El Mundo, 18-IX-1999.

Tudela. Olivia. "Xosé Carlos Caneiro: A nosa literatura ten poder e ten que seguir cara adiante". Eco 114 (2000): 66-67.

Velasco Oliaga, Javier. "Entrevista a Inma López Silva, autora de Los días iguales de cuando fuimos malas". Todo Literatura, 27-I-2017.

Recebido em: 25/03/2019

Aceito em: 07/06/2019

Publicado em: Setembro de 2019

Xosé Manuel Dasilva. E-mail: jdasilva@uvigo.es.

ORCID: https://orcid.org/0000-0003-1492-4549 